\title{
Developing a national research agenda to reduce consumption of sugar-sweetened beverages and increase safe water access and consumption among 0 - to 5-year-olds: a mixed methods approach
}

\author{
Emily W Duffy ${ }^{1, *}$, Megan M Lott' ${ }^{1}$ Emily J Johnson' and Mary T Story ${ }^{2}$ \\ ${ }^{1}$ Healthy Eating Research, Duke University, Duke Global Health Institute, 310 Trent Drive, Box 90519, Durham, \\ NC 27708 USA: ${ }^{2}$ Healthy Eating Research, Community \& Family Medicine and Global Health, Duke University, \\ 310 Trent Drive, Durham NC 27708 USA
}

Submitted 25 November 2018: Final revision received 8 April 2019: Accepted 3 May 2019: First published online 5 September 2019

\begin{abstract}
Objective: Sugar-sweetened beverage (SSB) consumption in early childhood is a public health concern. Adequate hydration in early childhood is also important. We developed a national research agenda to improve beverage consumption patterns among 0-5-year-olds. This article focuses on the process used to develop this research agenda.

Design: A mixed methods, multi-step process was used to develop the research agenda, including: (i) a scientific advisory committee; (ii) systematic reviews on strategies to reduce SSB consumption and increase water access and consumption; (iii) two stakeholder surveys to first identify and then rank strategies to reduce SSB consumption and increase water access and consumption; (iv) key informant interviews to better understand determinants of beverage consumption and strategies to improve beverage consumption patterns among high-risk groups; (v) an in-person convening with experts; and (vi) developing the final research agenda. Setting: This process included research and stakeholders from across the United States.

Participants: A total of 276 participants completed survey 1 and 182 participants completed survey 2. Key informant interviews were conducted with 12 stakeholders. Thirty experts attended the convening, representing academia, government, and non-profit sectors.

Results: Thirteen key issue areas and 59 research questions were developed. Priority topics were beverage consumption recommendations, fruit-flavoured drink consumption, interventions tailored to high-risk groups, and family engagement in childcare.

Conclusions: This research agenda lays the groundwork for research efforts to improve beverage patterns of young children. The methods used can be a template to develop research agendas for other public health issues.
\end{abstract}

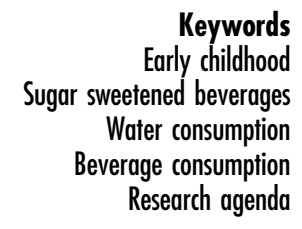

Keywords eetened beverages Research agenda
Despite current dietary guidance and risk of adverse health effects, many infants and young children in the United States consume sugar-sweetened beverages $(\mathrm{SSB})^{(1-3)}$. Racial/ethnic disparities in SSB consumption also exist, with black children being more likely to consume SSB than their white and Hispanic counterparts $^{(1)}$. At approximately six months, it is recommended that children begin drinking plain water. Maintaining adequate hydration through water consumption can support healthy taste preference development, cognition, and weight among young children ${ }^{(4-7)}$. However, $30-38 \%$ of $12-48-$ month-olds do not consume plain drinking water on a given day and inequities in access to safe drinking water in the United States have also been documented ${ }^{(3,8)}$. Furthermore, even in communities with safe drinking water, negative perceptions of tap water are pervasive and may be associated with SSB consumption ${ }^{(9,10)}$. 
Early childhood is a critical period for the development of lasting food preferences and dietary patterns ${ }^{(11,12)}$. To reduce SSB consumption among young children and promote health and well-being, dual strategies to decrease SSB consumption and increase water access and consumption are needed. Recent systematic reviews highlight the dearth of evidence on effective strategies to improve beverage consumption in this age group ${ }^{(13,14)}$. Given the lack of research and the pressing need to improve the beverage patterns of young children, an evidence-based research agenda to identify promising strategies and related research questions was warranted.

Research agendas have been developed for a variety of public health issues and serve as an effective means for informing funders, advocates and researchers about priority topics for future work and for coordinating research efforts among these groups ${ }^{(15-17)}$. Healthy Eating Research (HER), a national programme of the Robert Wood Johnson Foundation, developed a national research agenda to reduce SSB consumption and increase safe water access and consumption among 0 - to 5-year-olds in the United States, particularly those at greatest risk of poor health and dietary outcomes. However, without specificity, research agendas often lack utility. In an effort to provide stakeholders with an actionable list of research priorities, this article puts forward not only broad thematic areas, but also specific, prioritized research questions within each thematic area that could be used as the basis for writing a grant, developing a request for proposals, or guiding organizational strategic planning.

\section{Research Agenda Scope}

HER supports research on policy, systems and environmental (PSE) strategies that have strong potential to promote healthy eating among children, especially among lower-income and racial/ethnic minority populations at risk of poor nutrition and obesity. PSE strategies modify settings where people live, learn, work and play. These strategies, such as calorie and nutrient labelling on menus or nutrition standards for food available in childcare facilities, go beyond programming to embed changes in communities, and are designed to be more sustainable and reach a larger number of people than programming alone. Given HER's exclusive focus on PSE strategies and the lack of research on PSE strategies among 0- to 5-year-olds, the research questions put forward here predominantly focus on PSE approaches to reduce SSB consumption and increase water access and consumption. SSB are defined here as any drink with added sugar. Water is defined as safe drinking water that could be obtained from a tap or bottled water.

Throughout the development of this research agenda, stakeholders identified many questions related to safe water access. We acknowledge safe water access is not universal; however, it is beyond the scope and expertise of HER as a research programme focused on nutrition to address issues such as infrastructure, plumbing and water remediation. We outline a series of research questions related to safe water access and acknowledge that future efforts in this field will require partnerships between nutrition and environmental health stakeholders. Finally, given the disparities in SSB consumption and water access and consumption across racial, ethnic, geographic and socioeconomic groups, research gaps relevant to several racial/ethnic or geographic groups are highlighted in this research agenda. For the purposes of this research agenda, we focused on demographic groups that have the highest SSB consumption or lowest water access or consumption, subsequently referred to as 'priority populations'. Priority populations are groups that experience the greatest nutrition and weight-related health inequities, and include: Non-Hispanic black, Hispanic/Latino, Asian American/ Native Hawaiian/Pacific Islander, American Indian/ Alaska Native, and rural settings.

The purpose of this article is to present the research agenda and describe the rigorous, multi-step process used. This agenda can inform funders' and researchers' future efforts. Furthermore, the process used to create the agenda may be a useful model for other research topics.

\section{Methods}

To develop this research agenda, HER used a structured, mixed methods approach and completed the following six steps over a 6-month period (December 2017-June 2018): (1) convened a scientific advisory committee; (2) commissioned systematic literature reviews on strategies to reduce SSB consumption and strategies to increase drinking water access and consumption among 0 - to 5-year-olds; (3) conducted two sequential stakeholder surveys to (a) collect innovative strategies to eliminate SSB consumption and promote water access and consumption, and (b) rank the strategies identified in the systematic reviews and survey 1 on various dimensions; (4) conducted key informant interviews with representatives from priority populations; (5) held an in-person convening with experts; (6) developed the research agenda based on Steps 1-5. The steps in this process were informed by the learnings from the prior steps, with the exception of the scientific advisory committee which informed every step of the process. This process is outlined in Fig. 1 and described below. This process builds on methods used by others to develop research agendas in recent years ${ }^{(15-17)}$.

\section{Step 1. Scientific advisory committee}

HER formed a diverse scientific advisory committee to provide input on the plan for developing the research agenda, the methodology used in the systematic reviews and stakeholder surveys, the content of the surveys, the agenda for the convening, and the final content of the research agenda. This committee consisted of 11 researchers 


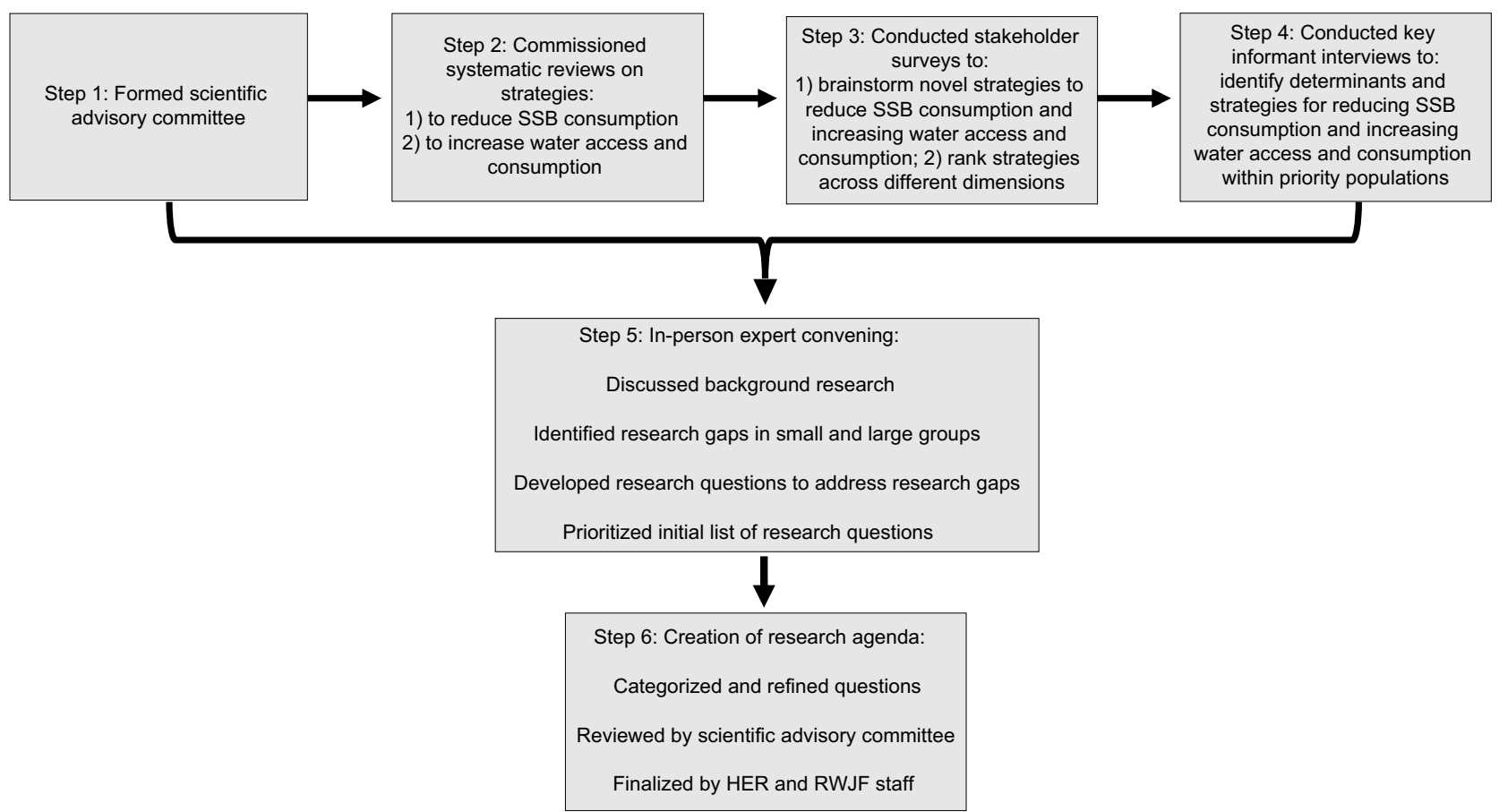

Fig. 1 Process for developing the national research agenda

with a range of expertise related to early childhood nutrition, strategies to improve beverage consumption in early childhood, and our priority populations.

\section{Step 2. Systematic reviews}

HER commissioned two systematic reviews of peerreviewed literature to identify effective strategies for reducing SSB consumption and increasing water access and consumption among 0- to 5-year-olds and priorities for future research efforts. One review focused on strategies to reduce SSB consumption and the other review focused on strategies to increase water access and consumption $^{(13,14)}$. The effective strategies identified in these systematic reviews, in addition to some of the strategies from the stakeholder survey 1, were then ranked in the stakeholder survey 2 .

\section{Step 3. Stakeholder surveys}

In addition to identifying strategies to be ranked in stakeholder survey 2 , the systematic reviews also highlighted gaps in the literature of evaluations of specific types of strategies for improving beverage consumption among 0 - to 5-year-olds. For example, very few of the studies identified in the reviews examined the impact of PSE strategies and no studies focused on water safety. To understand what some of these potentially promising but not yet evaluated strategies may be, HER sent an online survey to researchers, advocates, and other individuals across the country working in the areas of SSB reduction or water access and promotion.
We identified more than 300 individuals working on these issues using a snowball sampling approach and sent the survey via email. We augmented this list by sending the survey to 13 email mailing lists with a focus on SSB or water. In this first stakeholder survey, respondents were asked to brainstorm and list up to six (three SSB and three water) innovative and novel strategies that they thought could make an important impact on both eliminating SSB consumption and promoting water access and consumption among 0- to 5-year-olds (see Supplemental File 1 for full survey text). These responses were analysed in NVivo Version 11 (QSR International Pty Ltd, 2018) for emergent themes, sub-themes, and target settings. The scientific advisory committee reviewed the key themes from stakeholder survey 1 and selected 10 SSB strategies and 15 water strategies that were then ranked, in addition to the effective strategies from the systematic reviews in stakeholder survey 2 .

We then conducted the second, follow-up online stakeholder survey, where respondents ranked the effective strategies from the systematic reviews (Step 2) and the novel and innovative strategies identified in the survey 1 on five domains on a Likert scale from 1 (lowest) to 5 (highest) based on their: feasibility, effectiveness, reach, impact on health equity and overall importance. These domains are described in Table 1, and the definitions were developed by the scientific advisory committee. Table 2 lists the strategies identified in the systematic reviews and survey 1 that were ranked in survey 2 . The full survey 2 content can be found in Supplemental File 1. We sent survey 2 to the same individuals and mailing lists as survey 1 . Some 
Research agenda to improve beverage intake

Table 1 Definitions of the domains used in the survey 2 to evaluate strategies

\begin{tabular}{ll}
\hline Domain & \multicolumn{1}{c}{ Definition $^{1}$} \\
\hline $\begin{array}{l}\text { Overall importance } \\
\text { Feasibility }\end{array}$ & $\begin{array}{l}\text { Overall importance of the strategy for reducing SSB consumption or increasing water consumption/access. } \\
\text { How difficult it would be to implement the strategy, accounting for political challenges, legal considerations, }\end{array}$ \\
Effectiveness & economic factors, industry opposition, technical obstacles etc. \\
Reach & How much a strategy would reduce SSB consumption or increase water consumption/access. \\
Health equity & $\begin{array}{l}\text { Whether large numbers of children would be touched by the strategy. } \\
\text { How much a strategy would reduce existing disparities in SSB consumption or water consumption/access } \\
\text { (i.e. disparities among race, ethnicity, socioeconomic status, and geographic location). }\end{array}$
\end{tabular}

${ }^{1}$ These definitions were developed with the input of the scientific advisory committee.

individuals completed both survey 1 and 2, but it was not required for participants to complete both.

\section{Step 4. Key informant interviews}

One of the goals of this research agenda was to identify effective strategies and corresponding research gaps for reducing SSB consumption and increasing water access and consumption among the priority populations for this project. However, few of the studies identified through the systematic reviews (Step 2) evaluated the impact of strategies on these five demographic groups. Additionally, the stakeholder surveys (Step 3) asked participants to rank strategies based on potential impact on health equity, but did not ask detailed questions about which strategies may be most effective among particular population groups. Thus, HER staff conducted 12 key informant interviews with researchers and practitioners with expertise or experience working with our priority population groups to fill in the gaps in research among these demographic groups highlighted by Steps 2 and 3. The interview guide asked about determinants of SSB consumption, water consumption and water access among 0-5-year-olds, potentially promising strategies to reduce SSB consumption and increase water access and consumption among 0-5-year-olds, and key research questions related to healthy beverage consumption in early childhood. All questions were framed so answers were specific to the demographic group of interest. We identified the key informants with the input of the scientific advisory committee members, many of whom had extensive expertise related to at least one of our priority population groups.

Two or three representatives were interviewed from each priority population. The findings of these interviews are by no means generalizable; however, we presented the themes from the interviews at the in-person meeting (Step 5) and they informed convening attendees' knowledge of the strategies that may hold promise for shifting beverage consumption patterns in priority populations, and questions for the research agenda that may be relevant to the priority populations.

\section{Step 5. In-person convening with experts}

The goals of the in-person convening were to: share the findings from Steps 2-4 (the systematic reviews, stakeholder surveys, and key informant interviews); use the promising strategies identified through Steps 2-4 to develop research questions; and then prioritize research gaps identified by convening participants. The convening was held in Washington, DC on 17 and 18 May 2018. The event was organized and facilitated by HER staff, and 30 experts from academic, non-profit, government, advocacy, and philanthropic organizations participated in the meeting. The agenda for the convening can be found in Supplemental File 2.

We used the first day of the convening to present background research findings from Steps 2-4, consider if key strategies to reduce SSB consumption or increase water access or consumption were missing from this background research, and begin to consider some key research gaps based on the background research presented. On the second day, attendees participated in three structured small group discussions to create research questions on a variety of topics.

First, we divided meeting participants into small groups based on strategy type (e.g. price changes, changes in physical access) and they identified research gaps related to their group's strategy type. Next, we divided attendees into small groups by setting (e.g. policy/systems, industry, childcare) and asked them to identify additional research gaps related to their assigned setting. After these two discussions, attendees reviewed the research questions that had been developed based on the first two activities and participated in the third small-group exercise to identify gaps in the current set of questions related to health equity and priority populations and develop additional research questions to address these gaps.

Throughout the small group discussions, we asked participants to reference the findings from Steps 2-4 to ensure the research questions addressed gaps identified by the background research. Attendees developed all research questions in the small group discussions with the following criteria in mind - research questions should focus on: (i) reducing consumption of SSB and/or increasing access to and consumption of safe water among 0 - to 5year-olds; (ii) PSE strategies or a combination of PSE and individual-level strategies; (iii) strategies that have the potential to eliminate inequities (i.e. differences/disparities that are avoidable and unjust) in SSB consumption and 
Table 2 Strategies to reduce SSB consumption and increase water access and consumption ranked in survey 2

Strategies to reduce SSB consumption

\section{Education}

Train providers to screen children for SSB consumption and educate parents on reducing consumption during office visits

Provide counselling to parents/caregivers in healthcare settings to reduce SSB consumption, often using behaviour change techniques

Educate children on the importance of eliminating SSB using popular, culturally appropriate TV shows and radio, or celebrity endorsement of healthy beverages to target parents

Educate pregnant mothers to reduce their SSB consumption during pregnancy and to restrict SSB for the child

Teach children and parents/caregivers about reducing SSB consumption in non-clinical settings, often using behaviour change techniques

Leverage technology targeting children and parents/caregivers to reduce SSB consumption

Teach children and parents/caregivers about reducing SSB consumption using passive education methods

\section{Public awareness campaigns and contests}

Launch public awareness media campaigns to educate communities on the importance of reducing SSB consumption using various media outlets

Organize campaigns, challenges, and contests to reduce SSB consumption in child/daycare settings

\section{Marketing and advertising changes}

Modify advertising and promotion of SSB

Eliminate increased marketing for SSB during days of the month when Supplemental Nutrition Assistance Program (SNAP) benefits are issued

\section{Price changes}

Make SSBs more expensive

\section{Reduce physical access to SSB}

Prohibit serving, marketing, or selling SSB in any childcare centre, school, or government agency receiving or distributing federal funds

Prohibit all licensed Early Care and Education homes and centres (not just federally funded) from serving SSB to children as part of licensing regulations

Prohibit purchase of SSB with SNAP benefits

Prohibit the sale of SSB within a certain radius of child-oriented settings, schools, government facilities, public spaces to reduce density of SSB vendors

\section{Labelling and packaging of SSB}

Change SSB labelling and packaging to deter purchasing/consumption

\section{Improve setting and programme capacity}

Train providers and improve setting capacity to reduce SSB consumption

Establish a recognition or star rating programme for any organization that doesn't serve SSB, and make this easily accessible via an online platform

Strategies to increase access to/consumption of safe drinking water

\section{Education}

Educate providers who work with children on importance of water consumption

Teach children and parents about increasing water consumption using in-person education sessions

Leverage technology to educate children and parents on water consumption

Teach children and parents/caregivers about increasing water consumption using passive education methods

Develop a peer-to-peer support group for exchange of information related to water consumption for parents/caregivers

\section{Public awareness campaigns and contests}

Launch public awareness media campaigns to educate communities on the importance of drinking water

Use games or competitions to make water consumption fun and enjoyable for children

\section{Marketing and advertising changes}

Use popular, culturally appropriate cartoon characters or celebrities to promote water consumption among children Increase visibility of water in the food/beverage retail environment to increase purchasing and consumption 
Research agenda to improve beverage intake

Table 2 Continued

Strategies to increase access to/consumption of safe drinking water

Price changes

Decrease price of water relative to SSB

Promote water consumption among WIC and SNAP participants by providing greater benefit allotments for water purchases

Increasing physical access to water

Increase the availability of safe drinking water

Make water the default beverage choice (over SSB) at places where children are being served meals

Implement policy specifying the types of beverages served and provided to children in Care and Education sites

Create a program that sends safe drinking water home with children, especially in areas with known unsafe water sources

Provision and modification of drinking vessels

Increase the intake of water through the provision of drinking vessels that are developmentally appropriate for young children

Promote water consumption with fun/attractive designs on self-serve water stations and vessels

Water quality

Provide resources, technical assistance and training for water quality testing, communication and remediation

Infuse water with fruit in order to improve taste and palatability for children

Improve setting and programme capacity

Organizational strategies to promote water consumption in schools, government facilities, and public spaces

water access and consumption; and (iv) being specific and actionable.

HER staff wrote each of the research questions developed during small group discussions on a sticky note. Once all research questions from the small group discussions were refined and listed on sticky notes, we then distributed the sticky notes throughout the meeting room and asked participants to work through a two-part prioritization activity. First, we asked each participant to place dots on the six research questions they felt were the most important to address in the next five years. Next, we gave each participant four pieces of white paper and one piece of yellow paper on which we asked them to vote for their top five research questions in terms of overall importance. The yellow piece of paper was used to vote for the one question participants felt was most important to be included in the resulting research agenda (overall most important), while the other four white slips of paper were of equal weight (second-fifth most important).

\section{Step 6. Development of research agenda}

All convening proceedings, including group discussions, research questions developed or identified, and votes from the prioritization activities were recorded by six note takers trained in nutrition and research methods. Following the convening, two people (EBW and EJJ) thematically categorized research questions that were generated and ranked during day two of the convening as well as questions that were discussed in small groups. We removed duplicate questions and combined similar questions. We excluded some questions for reasons such as being out of scope, too specific, or not sufficiently refined. EBW and EJJ cross-checked the top-ranking strategies identified in the stakeholder surveys with the resulting list of research questions to ensure that all high-ranking strategies identified in the stakeholder surveys were represented in the research agenda. We then circulated the research agenda to the scientific advisory committee for their review and feedback. The research agenda produced as a result of this process can be found in Supplemental Table 1.

\section{Results}

\section{SSB consumption systematic review findings}

The detailed findings of this systematic review are published elsewhere ${ }^{(13)}$ but, overall, the review found 27 studies focused on 0- to 5-year-olds and uncovered several strategies that were effective in reducing SSB consumption. Eighteen studies were randomized controlled trials, four were quasi-experimental studies, and five were pre-post evaluations. Most of the studies included targeted multiple behaviours such as beverage consumption, physical activity or sleep. The majority of interventions were educational, one study was a systems-level change and another involved an environmental change. Successful studies, defined as significantly decreasing SSB consumption among children for at least one follow-up and/or one type of SSB, used strategies such as: (i) education, including in-person individual and group education for parents and children or passive education through material distribution; (ii) technology, including using text messages; (iii) training for caregivers and healthcare providers; and (iv) changes in physical access to beverages. The authors concluded that the most successful interventions often 
focused on vulnerable groups, specifically targeted SSB consumption or oral health, were conducted in childcare and healthcare settings, used three or more strategies, and had higher levels of contact with the target population. The review identified few studies that evaluated PSE changes and pointed to the need for future research to evaluate PSE changes and examine strategies implemented across multiple settings and populations. We included the successful strategies identified by this review in the list of strategies in stakeholder survey 2 . We also discussed the research gaps highlighted by this review at the in-person meeting and they informed the questions in the research agenda.

\section{Drinking water access and consumption systematic review findings}

The detailed findings of this review are also described elsewhere ${ }^{(14)}$. This review examined strategies to increase water access and consumption among 0- to 5-year-olds. Twenty-five articles met the inclusion criteria, 19 of which were deemed effective or promising (meaning they had at least one significant result at any follow-up). These studies took place in a variety of settings including childcare, social service agencies, homes, and afterschool programmes. There were no studies in clinical settings. Fifteen studies used a randomized study design, the remaining studies used quasi-experimental designs. The strategies most commonly evaluated were increasing water access and convenience, policy and practice changes, and providing education or training for caregivers. Only three of the 25 included studies targeted water access or consumption independently; most interventions targeted other nutrition or physical activity behaviours in addition to water consumption. Additionally, none of the studies provided information about the quality of water provided to children or families. We included the effective strategies identified in this review in the list of strategies in stakeholder survey 2. We also discussed the research gaps highlighted by this review at the in-person meeting and they informed the questions in the research agenda.

\section{Stakebolder survey findings}

For the stakeholder survey 1, there were 276 respondents, generating 676 SSB strategies and 593 water strategies. Many similar themes emerged in the responses. The most common themes for SSB strategies were: modifying physical access to SSB (34.3\% of responses), education $(28.7 \%)$, and price changes (90\%). The most common themes for water strategies were: modifying physical access to water (48.2\%), education $(32.5 \%)$, addressing water quality and safety $(16 \cdot 7 \%)$. Detailed findings from this survey can be found elsewhere ${ }^{(18)}$.

The number of responses for survey 2 was slightly lower, with 182 people completing the survey. In the second survey, the five highest ranking strategies to reduce SSB consumption in terms of overall importance scores were: (i) prohibiting serving, marketing or selling SSB in childcare settings receiving federal funds; (ii) making SSB more expensive; (iii) modifying advertising and promotion of SSB; (iv) prohibiting all licensed childcare homes and centres from serving SSB to children as part of licensing regulations; and (v) training providers to screen children for SSB consumption and educate parents on reducing consumption during office visits. The five highest-ranking strategies to increase water access and consumption in terms of overall importance scores were: (i) increasing availability of safe drinking water; (ii) making water the default beverage choice at food retailers; (iii) implementing policy-specifying types of beverages that can be provided to children at early childcare centres; (iv) decreasing the price of water relative to SSB; and (v) organizational strategies to promote water consumption in schools, government facilities, and public spaces. More detailed findings from this survey can be found elsewhere ${ }^{(18)}$.

\section{Key informant interview findings}

Key themes from the interviews are summarized in Supplemental Tables 2 and 3. The content of these interviews has not yet been formally analysed as we are currently scaling this project up to increase generalizability and sample size. However, some of the key themes that emerged across all 12 interviews for SSB consumption determinants were: knowledge of the health impacts of SSB, lack of access to safe drinking water, poverty, and the appeal of SSBs over other beverage choices such as water. Consistent water access and consumption determinants included: poverty, the perception of tap water safety, physical properties of tap water such as taste or colour, and the cost of bottled water. Key informants across the five demographic groups raised several potentially promising strategies to reduce SSB consumption: educating parents and caregivers, providing consistent information about beverage recommendations in early childhood, and interventions in childcare. For water consumption and access, some commonly mentioned strategies included: providing access to water throughout the day in childcare settings, encouraging people to test their water supply, returning to indigenous or traditional food and beverage patterns, and implementing policies that provide water as the default beverage.

\section{Research agenda findings}

Based on the information gathered in Steps 2-5, 13 key issues emerged as priorities for future research efforts. Within these 13 key issues, stakeholders at the in-person convening identified 59 unique research questions. The priority research questions (defined here as one of the 10 highest scoring questions in terms of total number of votes at the in-person meeting for at least one of the three prioritization categories used) can be found in Table 3. The 
Table 3 Priority research questions in the national research agenda by key issue area

\begin{tabular}{lcc}
\hline Key issue area & $\begin{array}{c}\text { Important to address } \\
\text { in next 5 years }\end{array}$ & $\begin{array}{c}\text { Overall most } \\
\text { important }^{2}\end{array}$ \\
$\begin{array}{c}\text { 2nd-5th most } \\
\text { important }^{3}\end{array}$ \\
\hline
\end{tabular}

Measures of consumption and baseline understanding of consumption patterns

1. What are the beverage consumption patterns of $0-5$-year-olds and how do

these consumption patterns differ by setting and among priority populations?

$\mathrm{X}$

$\mathrm{X}$

Beverages in the food retail environment

2. What mix of price, promotion, placement, including check-out or other instore marketing, would be effective in shifting the mix of beverages sold (and consumed) to decrease SSB and increase water consumption among 0-5 year olds, especially for priority populations?

3. What is the comparative effectiveness of various modifications to the SNAP programme (retailer standards, SSB restrictions, healthy beverage incentives) in improving beverage patterns of $0-5$-year-olds?

\section{Recommendations for beverage consumption in early childhood}

4. What are the evidence-based, culturally-appropriate recommendations for water, SSB, milk and flavoured milk, and $100 \%$ juice intake for children from birth to age 5 ? How can these recommendations, once developed, be disseminated effectively?

5 . What are current healthcare provider practices regarding recommendations to parents and caregivers about water safety, access, and consumption? And what are effective methods to ensure healthcare providers are providing consistent messages?

\section{Retail price of SSB and water}

6 . What is the influence of SSB taxes on parent/caregiver purchases, beverage intake, and health outcomes among $0-5$-year-olds from priority populations and their families?

\section{Beverage substitution}

7. What are culturally-appropriate, healthy substitutes for SSB among priority populations? Does promoting water as the only healthy substitute alienate certain high-risk groups?

\section{Fruit-flavoured drinks and artificially sweetened drinks}

8. What aspects of product packaging of fruit-flavoured drinks, including nutrition claims, images, or shape, mislead parents to believe these are healthy options for children? How do these elements of product packaging affect product purchases? What is the impact of using the same techniques that are used to promote fruit-flavoured drinks to instead encourage purchase of water? 9. How do perceptions of fruit-flavoured drinks and packaging (nutrient claims, images, shapes) differ between priority populations? How does an individual's country of origin affect these perceptions?

\section{Healthcare interventions to improve beverage consumption patterns}

10 . What is the impact of education/counselling in paediatric oral healthcare settings about SSB and water consumption on beverage patterns of 0-5-yearolds and what are best practices for counselling in this setting?

11. What guidance are healthcare providers currently providing pregnant women regarding beverage consumption? What is the impact of a pre-natal healthy beverage educational programme provided in a clinical setting (i.e. by $\mathrm{OB} / \mathrm{GYNs}$, nurse practitioners, nurse midwives) on maternal and infant beverage intake patterns and health outcomes?

\section{Access to safe water and tap water perception}

12. What is the impact of integrating a water security screening questionnaire into the Electronic Health Record and designing appropriate interventions for water insecure individuals on water intake, other beverage intake, and health outcomes among $0-5$-year-olds?

Achieving equity in beverage consumption patterns and addressing the social determinants of health

13. Among priority populations, what are the determinants of and attitudes about SSB, $100 \%$ juice, water, and artificially-sweetened beverage consumption? What is the effectiveness of evidence-based strategies to reduce SSB consumption and increase water access and consumption in priority populations? 


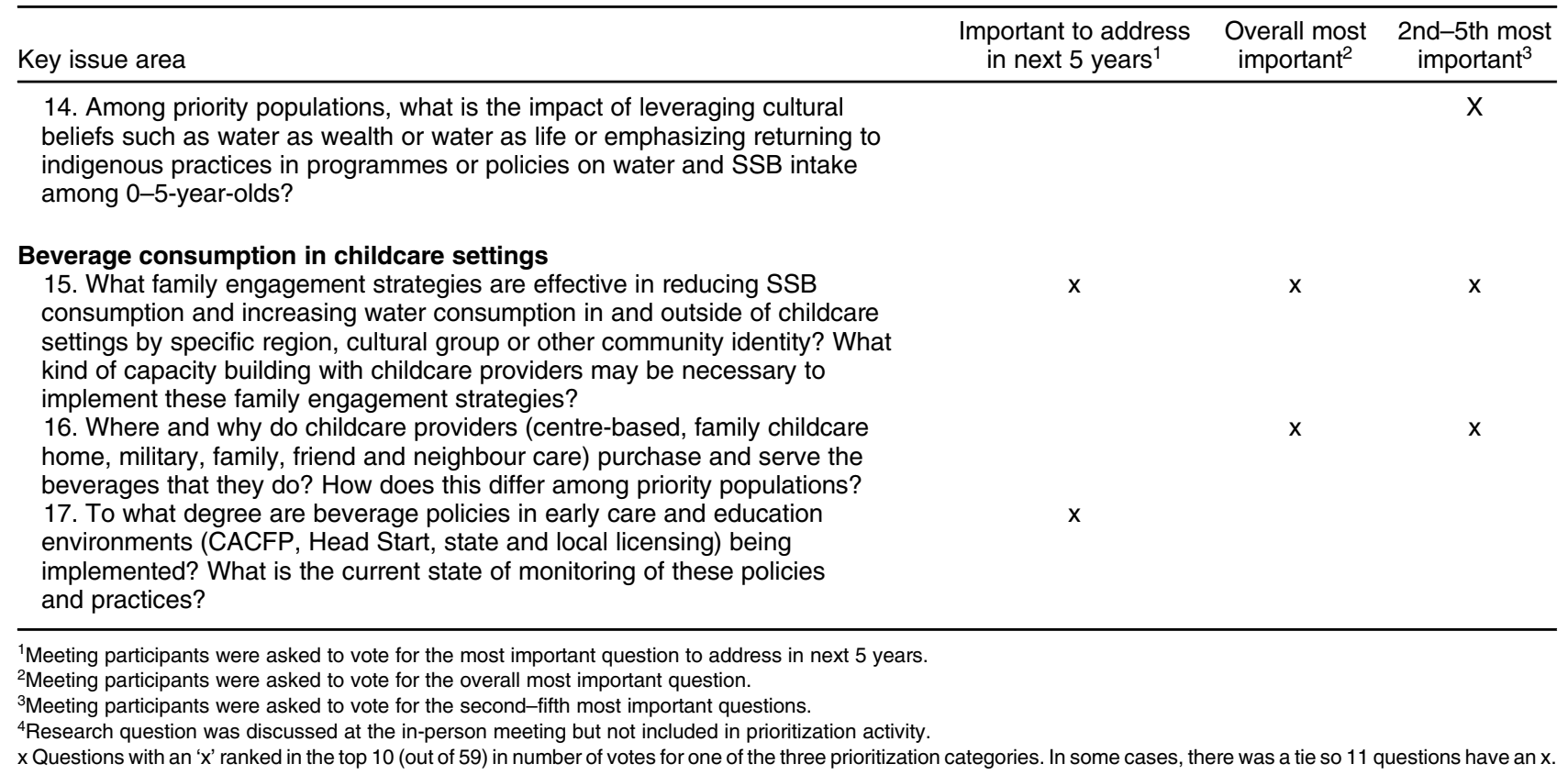

complete list of 59 research questions can be found in Supplemental Table 1.

Below are the research questions that ranked in the top 10 for all three categories used in the prioritization activities (most important to answer in the next 5 years, second-fifth most important research questions, and overall most important research question):

- What are the evidence-based, culturally-appropriate recommendations for water, SSB, milk and flavoured milk, and $100 \%$ juice intake for children from birth to age 5 ? How can these recommendations, once developed, be disseminated effectively?

- What aspects of product packaging of fruit-flavoured drinks, including nutrition claims, images, or shape, mislead parents to believe these are healthy options for children? How do these elements of product packaging affect product purchases? What is the impact of using the same techniques that are used to promote fruit-flavoured drinks to instead encourage purchase of water?

- How do perceptions of fruit-flavoured drinks and packaging (nutrient claims, images, shapes) differ between priority populations? How does an individual's country of origin affect these perceptions?

- Among priority populations, what are the determinants of and attitudes about SSB, $100 \%$ juice, water, and artificially-sweetened beverage consumption? What is the effectiveness of evidence-based strategies to reduce SSB consumption and increase water access and consumption in priority populations?

- What family engagement strategies are effective in reducing SSB consumption and increasing water consumption in and outside of childcare settings by specific region, cultural group or other community identity? What kind of capacity building with childcare providers may be necessary to implement these family engagement strategies?

One of the goals of this research agenda was to identify research gaps that were relevant to our priority populations. It was not possible to present research questions tailored to each demographic group given the dearth of existing research; however, many of the key issue areas highlighted in the full research agenda (Supplemental Table 1) present research priorities that were cross-cutting across all priority populations.

\section{Discussion}

This study used a rigorous, mixed-methods approach to develop a national research agenda to reduce SSB consumption and promote water access and consumption among 0- to 5-year-olds. No such research agenda with priorities highlighted exists, to our knowledge. Thirteen key issue areas and 59 research questions were developed. Topics that emerged as priorities were beverage consumption recommendations, fruit-flavoured drink consumption, interventions to improve beverage consumption patterns tailored to high-risk groups, and family engagement in childcare. The specific questions outlined by this research agenda can serve as the foundation for future requests for proposals from funding agencies, research grant proposals, and strategic planning activities for organizations working on these topics. As a direct result of this research agenda, HER released a special call for proposals specifically on beverage consumption in early childhood. Additionally, 
the process detailed here can be applied by other researchers, coalitions, or funders to develop similar research agendas on different public health topics.

The systematic reviews identified critical research gaps that can be leveraged in future efforts to improve beverage consumption patterns among infants and young children. For example, both the water and SSB reviews pointed to the need for evaluation of more PSE interventions to improve beverage intake in this population. The stakeholder surveys as well as the key informant interviews illuminated a wide variety of strategies that may hold promise to improve beverage consumption patterns of infants and young children, particularly those at greatest risk of poor dietary intake. While some of these strategies such as taxation or healthy beverage defaults have been evaluated at the population-level or among older children, future research efforts should aim to understand the impact of these interventions on young children. Many of the strategies mentioned in the key informant interviews have not been evaluated, to our knowledge, and provide exciting ideas for future research endeavours.

The prioritization process at the convening revealed several key research priority areas: evidence-based recommendations for beverage intake in early childhood, fruitflavoured drink marketing and labelling, determinants of beverage consumption and effective strategies to improve beverage consumption among our priority populations, and family engagement strategies in early care and education settings.

Various scientific entities have put forth recommendations related to beverage consumption among 0 - to 5-year-olds ${ }^{(19-21)}$. However, there is currently limited or no guidance from authoritative bodies on some beverage types, such as beverages with non-nutritive sweeteners for 0-5-year-olds and some recommendations are contradictory or inconsistent. Stakeholders at the in-person meeting felt that addressing these gaps and inconsistencies was a top priority. They also discussed that specific, quantitative recommendations for plain drinking water are especially needed in this age group. Once these recommendations are established, efforts to consistently communicate the recommendations in ways that are culturally-relevant to diverse communities will be needed. The inclusion of children from birth to age two in the 2020 Dietary Guidelines for Americans will be a critical step toward establishing consistent recommendations for early childhood.

Experts also raised several questions about fruitflavoured drink consumption and marketing as top priorities. Fruit-flavoured drinks are the most commonly consumed variety of SSB among 0- to 5-year-olds. There are significant racial/ethnic disparities in the consumption of these beverages among young children with Black children being more likely to consume fruit-flavoured drinks than their White and Hispanic counterparts. These beverages often include health and nutrition claims such as 'contains $100 \%$ of Daily
Value of Vitamin C' on their packaging, which can be confusing to parents and caregivers, and fruit drinks geared toward children are more likely to have these claims than other fruit drinks ${ }^{(22)}$. Research specifically examining the impact of these claims on purchasing patterns is critically needed and could inform federal policy changes.

Racial/ethnic as well as income-based inequities in SSB intake and water access and consumption are a pressing public health problem. The stakeholders at the in-person convening felt that more research attention and funding should be devoted to identifying (i) the determinants of beverage consumption among high-risk groups and (ii) effective strategies to improve beverage consumption patterns.

Childcare settings are critical for improving healthy eating behaviours of infants and young children. Both systematic reviews developed as part of this project identified successful strategies in childcare settings. However, in order for these positive health behaviours to translate to the home setting and create sustainable change, we need to better understand effective strategies for childcare providers to engage families in improving beverage consumption behaviours. Including family engagement components in childcare interventions has been found to significantly improve the impact of the intervention on health outcomes such as child weight, so future research should examine the impact on other key health outcomes such as beverage consumption $^{(23)}$.

\section{Strengths and limitations}

There are limitations to the approach used to develop this research agenda. The convenience samples used in the stakeholder survey and the in-person convening may have been subject to biases that are inherent in research and could have impacted the content of the research agenda. For example, there were several key stakeholder groups that were not represented in the survey respondents (e.g. the food and beverage industry) and the survey results were likely impacted by self-selection bias. Also, a more extensive research question prioritization process with more stakeholders was not conducted following the convening due to timing issues. Finally, we were unable to present research questions specific to each of our priority populations and instead present research gaps that apply to many if not all of our priority populations. In the process of expanding our key informant interviews, we hope to address some of these gaps and present more specific research gaps for each demographic group. There are many strengths to the approach we used to develop this research agenda. We used a rigorous, multi-step 6 month process to arrive at the research questions listed in Supplemental Table 1. We also engaged a much greater number of sectors and individuals in the prioritization and development of strategies that informed the research 
agenda than previous research agenda processes have achieved. We feel the methods and process used to develop this research agenda could be applied to the development of future research agendas related to other public health issues.

\section{Conclusions}

The prevalence of SSB consumption and inadequate access to and consumption of water among 0 - to 5 -year-olds in the US are cause for concern, as are the racial/ethnic disparities in intake. We describe a mixed methods approach to develop a national research agenda to reduce SSB consumption and promote water access among young children aged 0 to 5 , and the resulting research questions were identified as most important to address. By detailing the methods used to develop the research agenda, we have provided a template for other groups wishing to undertake similar processes to address other public health issues. Answering key questions identified in this research agenda through rigorous research has the potential to impact beverage consumption patterns, and ultimately the health and well-being, of young children.

\section{Acknowledgements}

Acknowledgements: We would like to thank the members of the scientific advisory committee for the input and expertise that they provided throughout this process as well as members of the research teams that conducted the systematic reviews and stakeholder surveys. We would also like to thank the HER Team, our RWJF Program Officers who were engaged in supporting this project from conceptualization to publication, and the individuals that participated in the key informant interviews. Financial support: This work was supported by the Robert Wood Johnson Foundation (grant number 75169). Conflicts of interest: None. Authorship: E.W., M.S., and M.L. designed the research. E.W., M.L., and M.S. managed the research agenda setting process, research teams, and scientific advisory committee. E.W. and E.J. reviewed and analysed all research questions generated at the convening, and wrote the manuscript. All authors read, commented on, and approved the final manuscript. Ethics of buman subject participation: The key informant interviews were deemed non-human subjects research and exempt from further IRB Review by Duke Medicine's Institutional Review Board for Clinical Investigators (Protocol ID: Pro00093446).

\section{Supplementary material}

To view supplementary material for this article, please visit https://doi.org/10.1017/S1368980019002106.

\section{References}

1. Welker EB, Jacquier EF, Catellier DJ et al. (2018) Room for improvement remains in food consumption patterns of young children aged 2-4 years. J Nutr 148, 1536S-1546S. doi: $10.1093 / \mathrm{jn} / \mathrm{nxx} 053$.

2. Grimes CA, Szymlek-Gay EA \& Nicklas TA (2017) Beverage consumption among U.S. children aged 0-24 months: National Health and Nutrition Examination Survey (NHANES). Nutrients 9, 264. doi: 10.3390/nu9030264.

3. Kay MC, Welker EB, Jacquier EF et al. (2018) Beverage consumption patterns among infants and young children (0-47.9 months): data from the Feeding Infants and Toddlers Study, 2016. Nutrients 10, 825. doi: 10.3390/nu10070825.

4. Grandjean AC \& Grandjean NR (2007) Dehydration and cognitive performance. J Am Coll Nutr 26, 549S-554S.

5. Muckelbauer R, Libuda L, Clausen K et al. (2009) Promotion and provision of drinking water in schools for overweight prevention: randomized, controlled cluster trial. Pediatrics 123, e661-e667.

6. Lieberman HR (2007) Hydration and cognition: a critical review and recommendations for future research. $J \mathrm{Am}$ Coll Nutr 26 (suppl. 5), 555S-561S.

7. Mennella JA (2014) Ontogeny of taste preferences: basic biology and implications for health. Am J Clin Nutr 99, 704S-711S.

8. Balazs CL \& Ray I (2014) The drinking water disparities framework: on the origins and persistence of inequities in exposure. Am J Public Health 104, 603-611. doi: 10.2105/ AJPH.2013.301664.

9. Pierce G \& Gonzalez S (2017) Mistrust at the tap? Factors contributing to public drinking water (mis)perception across US households. Water Policy 19, 1-12. doi: 10.2166/wp. 2016.143.

10. Onufrak SJ, Park S, Sharkey JR et al. (2014) The relationship of perceptions of tap water safety with intake of sugar-sweetened beverages and plain water among US adults. Public Health Nutr 17, 179-185. doi: 10.1017/S1368980012004600.

11. Reidy KC, Deming DM, Briefel RR et al. (2017) Early development of dietary patterns: transitions in the contribution of food groups to total energy-Feeding Infants and Toddlers Study, 2008. BMC Nutr 3. doi: 10.1186/s40795-016-0124-0.

12. Birch LL \& Doub AE (2014) Learning to eat: birth to age 2 y. Am J Clin Nutr 99, 723s-728s. doi: 10.3945/ajcn.113.069047.

13. Vercammen KA, Frelier JM, Lowery CM et al. (2018) A systematic review of strategies to reduce sugar-sweetened beverage consumption among 0- to 5-year-olds. Obes Rev 19, 1-21. doi: 10.1111/obr.12741.

14. Cradock AL, Poole MK, Agnew K et al. (2019) A systematic review of strategies to increase drinking water access and consumption among 0- to 5-year-olds. Obes Rev June; doi: 10.1111/obr.12833. [Epub ahead of print] Review. PubMed PMID: 31250960.

15. Johnson DB, Quinn E, Sitaker M et al. (2014) Developing an agenda for research about policies to improve access to healthy foods in rural communities: a concept mapping study. BMC Public Health 14. doi: 10.1186/1471-2458-14-592.

16. Scutchfield FD, Perez DJ, Monroe JA et al. (2012) New public health services and systems research agenda: directions for the next decade. Am J Prev Med 42, S1-S5. doi: 10.1016/j. amepre.2012.01.027.

17. Brownson RC, Kelly CM, Eyler AA et al. (2008) Environmental and policy approaches for promoting physical activity in the United States: a research agenda.J Phys Act Health 5, 488-503.

18. Vercammen KA, Frelier JM, Lowery CM et al. (2018) Strategies to reduce sugar-sweetened beverage consumption and increase water access and intake among young children: perspectives from expert stakeholders. Public Health Nutr 21, 3440-3449. doi: 10.1017/S1368980018002604. 
19. US Department of Health and Human Services \& US Department of Agriculture (2015) 2015-2020 Dietary guidelines for Americans, 8th ed.

20. American Academy of Pediatrics Committee on Nutrition (2014) Pediatric Nutrition: Policy of the American Academy of Pediatrics. Elk Grove Village, Illinois: American Academy of Pediatrics.

21. Peérez-Escamilla R, Segura-Peérez S \& Lott M (2017) Feeding Guidelines for Infants and Young Toddlers: A Responsive Parenting Approach. Durham, North Carolina: Healthy
Eating Research. http://healthyeatingresearch.org (accessed June 2018).

22. Harris J, Schwartz M, LoDolce M et al. (2014) Sugary Drink FACTS 2014: Sugary Drink Marketing to Youth: Some Progress but Much Room for Improvement in Marketing to Youth. Hartford (CT): Rudd Center for Food Policy and Obesity.

23. Ward DS, Welker E, Choate A et al. (2017) Strength of obesity prevention interventions in early care and education settings: a systematic review. Prev Med 95, S37-S52. 